\title{
On the Stability of Cooperation Structures*
}

\author{
Guillaume Haeringer ${ }^{\dagger}$ \\ Departament d'Economia i d'Història Econòmica and CODE \\ Universitat Autònoma de Barcelona, Spain \\ November 22, 2000
}

\begin{abstract}
Qin (1996) recently showed that in a game of endogenous formation of cooperation structure, if the underlying TU-game is superadditive, then the full cooperation structure is stable. In this note, we characterize the class of games that ensure the stability of the full cooperation structure, and show that this class is much larger than that of superadditive TU-games.

KEYwords Cooperation structure, graph, Myerson value, stability. Journal of Economic Literature Classification: C71.

\footnotetext{
${ }^{*}$ I wish to thank Belén Jerez and Jordi Massó for incisive and helpful comments. Financial support from the European Commission, contract number HPMF-CT-1999-00284, is gratefully acknowledged.

${ }^{\dagger}$ Tel.: (+34) 9358125 05, Fax: (+34) 9358124 61, Departament d'Economia i d'Història Econòmica and CODE, 08193 Bellaterra (Barcelona), Spain. E-mail: ghaeringer@volcano.uab.es.
} 


\section{Introduction}

In a seminal paper, Aumann and Myerson (1988) proposed a model of endogenous cooperation structure formation. Cooperation structures represent situations in which players have the possibility to cooperate, or communicate, only with a subset of the population. An interesting feature of these situations is that two players who are in cooperation may not be in cooperation with the same subset of players. Hence, these structures may be represented by non-directed graphs. Aumann and Myerson proposed to use, in an extensive-form game, the Myerson value (Myerson, 1977) to compute players' payoffs in cooperation structures. A major drawback of their approach is that the order of play has to bet set exogenously. Recently, Qin (1996) reformulated Aumann and Myerson's game as a normal-form game. One of Qin's results is that if the underlying TU-game is superadditive, then the full cooperation structure (each player cooperates with all the other players) is stable in the sense that no player can profitably deviate by severing some of her links.

In this note we characterize the class of TU-games that admit a stable full cooperation structure, and show that it is much larger than the class of superadditive games. Indeed, we show that the full cooperation structure is stable if and only if the value of each player is individually rational and the TU-game is superadditive for two-player coalitions only. In particular, this implies that if, for some TU-game $(N, v)$ and two players $i$ and $j$, we have $v(\{i, j\})<v(\{i\})+v(\{j\})$, then the full cooperation structure is not stable.

The paper is organized as follows. In section 2 we present the framework. In section 3 we define the game of endogenous formation of cooperation structure, and state and prove our characterization result. 


\section{Definitions}

A transferable utility game (or a TU-game) is defined by a couple $(N, v)$ where $N=\{1, \ldots, n\}$ is the set of players, and $v$ is the characteristic function that maps the power set of $N$ to the set of real numbers (with the convention that $v(\varnothing) \equiv 0)$. The number $v(S)$ yields the worth of coalition $S \in 2^{N}$. The space of all TU-games with the set of players $N$ is denoted by $\Gamma_{N}$.

We assume that players are able to establish meaningful relationships with a subset of players, and we call the set of all private relationships a cooperation structure. A useful way to represent cooperation structures is by a non-directed graph, whose vertices represent players and whose edges represent the relationships that players have between each other.

We define a graph, $g$, as a set of links between players. We write $i: j \in g$ when $i$ and $j$ are linked in $g$. Two players $i$ and $j$ are indirectly linked in $g$ if there exists a sequence $i_{1}, \ldots, i_{k}$ in $N$, with $k \geq 3$, such that $i=i_{1}, i_{t-1}: i_{t}$ for all $t=2, \ldots, k$, and $i_{k}=j$. The sequence $i_{1}, \ldots, i_{k}$ is called a path. Two players are said to be connected if they are (indirectly) linked. A coalition $S$ is said to be connected if any pair of players $i, j \in S$ is connected by a path involving only players in $S$. We denote by $G R_{N}$ the set of all graphs and by $g_{N}$ the complete graph, i.e. the graph in which each player is linked with all other players,

$$
\begin{gathered}
g_{N}=\{i: j \mid i \in N, j \in N, i \neq j\}, \\
G R_{N}=\left\{g \mid g \subseteq g_{N}\right\} .
\end{gathered}
$$

For any graph $g, g \backslash\{i: j\}$ denotes the graph in which the link $i: j$ has been deleted, and $g \cup\{i: j\}$ is the graph to which the link $i: j$ has been added. We denote the graph $g$ restricted to the set of vertices $S$ by $g(S)$,

$$
g(S)=\{i: j \mid i, j \in S, i: j \in g\} .
$$

For any subset of players $S$, there is a unique partition of players that groups together players connected by $g(S)$. Such a partition is denoted 
by $S / g$. In other words, for any $i \in S$, the element of the partition $S / g$ containing $i$ also contains all other players that are connected to $i$ by $g(S)$. A formal definition of this partition is defined as follows,

$$
S / g=\{\{i \in S \mid i \text { and } j \text { are connected in } S \text { by } g\} \mid j \in S\} \text {. }
$$

\section{The game}

Qin's (1996) model works as follows. Given a TU-game $(N, v)$, we allow players to choose who they want to cooperate, or communicate with. Hence, each player can have private relationships only with a subset of the grand coalition. More formally, we define a non-cooperative game

$$
\left.\mathscr{G}_{\mu}^{(N, v)}=<N,\left(\Sigma_{i}\right)_{i \in N},\left(h_{i}\right)_{i \in N}\right)>
$$

where the TU-game $(N, v)$ is called the underlying game of $\mathscr{G}_{\mu}^{(N, v)}$. As in $(N, v)$, the set of players is $N$ and $\Sigma_{i}$ is the strategy space of player $i$. A strategy $\sigma_{i}$ for player $i$ is defined as a $n$-dimensional vector whose coordinates are either 0 or 1 . When $\sigma_{i}(j)=0$ player $i$ does not wish to form a link with player $j$, while he would be glad to create such a link when $\sigma_{i}(j)=1$. The $i$ th coordinate of $\sigma_{i}$ is assumed to be equal to zero, $\sigma_{i}(i) \equiv 0$. In other words, no player can make a link with herself. A link between players $i$ and $j$ is formed only if both $i$ and $j$ wish to create it: $\sigma_{i}(j)=\sigma_{j}(i)=1$. We denote the $n$-tuple of strategies by $\sigma=\left(\sigma_{1}, \ldots, \sigma_{n}\right)$. Each $\sigma \in\left(\Sigma_{i}\right)_{i \in N}$ gives a unique cooperation structure $g(\sigma)$,

$$
g(\sigma)=\left\{i: j \mid \sigma_{i}(j)=\sigma_{j}(i)=1\right\}
$$

For each cooperation structure $g$, players are rewarded using the Myerson value of the game $(N, v)$ on $g$, denoted by the $n$-dimensional vector $\mu(v, g)$.

The Myerson value is an allocation rule for TU-games with graphs. To compute the Myerson value for some game $(N, v)$ and some cooperation 
structure $g$, we first need to define a characteristic function embedded on graphs,

$$
\forall S \subseteq N, \quad(v / g)(S)=\sum_{T \in S / g} v(T)
$$

The Myerson value is the unique allocation $\mu$ rule that satisfies the Component Efficiency axiom,

$$
\sum_{i \in N} \mu_{i}(v, g)=\sum_{S \in N / g} v(N)
$$

and the Fairness axiom,

$$
\mu_{i}(v, g)-\mu_{i}(v, g \backslash i: j)=\mu_{j}(v, g)-\mu_{j}(v, g \backslash i: j), \quad \forall g \in G R_{N}, \forall i: j \in g
$$

Myerson proved that his value is closely related to the Shapley value. Indeed, we have

$$
\mu(v, g)=\varphi(v / g), \forall(N, v) \in \Gamma_{N}, \forall g \in G R_{N},
$$

where $\varphi$ is the Shapley value. It is straightforward to see that for the full cooperation structure, $v / g=v$, and hence $\mu\left(v, g_{N}\right)=\varphi(v)$. Clearly, if some player $i$ is not connected to anyone in the cooperation structure $g$, then $\mu_{i}(v, g)=v(\{i\})$. Note that it may happen that for some game $(N, v)$ and some $g$, we have $\mu_{i}(v, g)<v(\{i\})$.

Thus, the utility of a player in the game $\mathscr{G}_{\mu}^{(N, v)}$ under the strategy profile $\sigma$ is her Myerson value of the corresponding cooperation structure,

$$
h_{i}(\sigma) \equiv \mu_{i}(v, g(\sigma)) .
$$

Consider now a cooperation structure $g$ such that players $i$ and $j$ are not linked, i.e., $i: j \notin g$. Then, player $i$ desires to link up with player $j$ if and only if

$$
\mu_{i}(v, g \cup\{i: j\}) \geq \mu_{i}(v, g) .
$$


Given that the Myerson value satisfies the Fairness axiom, it is readily verified that if (5) holds true for player $i$, then it also holds true for player $j{ }^{1}$

A cooperation structure $g$ is stable in Qin's sense if there is a strategy profiles $\sigma$ such that $g(\sigma)=g$ and $\sigma$ is a Nash equilibria of the game $\mathscr{G}_{\mu}^{(N, v)}$. This result was also obtained in a similar framework by Dutta, van den Nouweland, and Tijs (1998). ${ }^{2}$ We show now that this result can be extended to a larger class of games.

Proposition 1 Let $(N, v)$ be a TU-game and $\mu$ the Myerson value. Then the full cooperation structure is stable if and only if $\mu_{i}\left(v, g_{N}\right) \geq v(\{i\})$ for all $i \in N$, and

$$
v(\{i, j\}) \geq v(\{i\})+v(\{j\}), \text { for all } i, j \in N .
$$

Proposition 1 says that if the Shapley value of each player in the game $(N, v)$ is individually rational then $g_{N}$ is stable if and only if the game $(N, v)$ is "superadditive" for two-player coalitions. Hence, the two conditions individual rationality and (6) - fully characterize the class of TU-games that ensure the stability of the full cooperation structure.

As the following example shows, superadditivity of the game $(N, v)$ is not necessary for the stability of the full cooperation structure.

Example 1 Consider the following $T U$ game $(N, v)$ with $N=\{a, b, c, d\}$,

\footnotetext{
${ }^{1}$ Reciprocity may not hold if player $i$ wants to link up with several players at a time.

${ }^{2}$ Dutta, van den Nouweland, and Tijs (1998) employed several equilibrium concepts, (undominated Nash equilibrium, coalition-proof Nash equilibrium and Strong Nash equilibrium) and did not use a specific solution concept to analyse to equilibria of the game.
} 
and $v$ defined by

$$
v(S)= \begin{cases}0 & \text { if }|S|=1, \\ 20 & \text { if }|S|=2, \\ 12 & \text { if }|S|=3 \\ 16 & \text { if }|S|=4 .\end{cases}
$$

The Myerson value of each player for the full cooperation structure is $\mu_{i}\left(v, g_{N}\right)=4$, for $i=a, b, c, d$, which is individually rational. Obviously, condition (6) is satisfied. Hence, Proposition 1 implies that the full cooperation structure is stable. Yet, it is obvious that the full cooperation structure is Pareto dominated, i.e. there is another cooperation structure (for instance $\left.g^{\prime}=\{a: b, c: d\}\right)$ that yields a strictly higher value to all players. ${ }^{3}$

In order to prove the proposition, we introduce more definitions.

For any cooperation structure $g$ and players $i$ and $j$ such that $i: j \in g$, define the set

$$
\mathbf{S}(g, i: j)=\{S \subseteq N \mid S / g=S \text { and } S /(g \backslash\{i: j\}) \neq S\}
$$

In other words, $\mathbf{S}(g, i: j)$ is the set of coalitions $S$ such that $S$ is connected in $g(S)$ but is non-connected in $g(S) \backslash\{i: j\}$. When no confusion is possible, we write $\mathbf{S}(i: j)$ instead of $\mathbf{S}(g, i: j)$. In other words, $\mathbf{S}(i: j)$ represents the set of coalitions $S$ that are connected thanks to $i: j$ when the graph is restricted to $S .{ }^{4}$ Thus, for any $S \in \mathbf{S}(i: j)$, if $i: j$ is deleted, $S /\left(g \backslash\{i: j\}=\left\{S_{i}, S_{j}\right\}\right)$, with $S_{i}$ and $S_{j}$ being the sets in $S /(g \backslash\{i: j\})$ that contain $i$ and $j$ respectively.

Proof of Proposition 1 Observe that there is a unique strategy profile $\sigma^{*}$ such that $g\left(\sigma^{*}\right)=g_{N}$. Hence, $\sigma^{*}$ is a Nash equilibrium if and only if

\footnotetext{
${ }^{3}$ A similar result was obtained by Jackson and Wolinsky (1996), who showed that some games $\mathscr{G}_{\mu}^{(N, v)}$ may have a stable cooperation structures that are not efficient, i.e. structures that do not maximize the worth of coalition(s).

${ }^{4}$ In graph terminology, we say that $\mathbf{S}(g, i: j)$ is the set of all subgraphs $\hat{g}$ of $g$ such that the link $i: j$ is critical in $\hat{g}$ - see Bollobás (1998).
} 
no player breaks one or more links. Hence, it suffices to compare, for any player $i$, her payoffs under the strategy profile $\sigma$ and under the strategy profile $\left(\sigma_{i}^{\prime}, \sigma_{-i}^{*}\right)$, where $\sigma_{i}^{\prime}(j)=0$ for at least one $j \in N \backslash\{i\}$.

Clearly, if the value of some player is not individually rational, then that player has an incentive to break up all her links (or any part thereof), and then the full cooperation structure is not stable. In other words, individual rationality is a necessary condition for the full cooperation structure to be stable. We now show that if the value of each player is individual rational, then stability is equivalent to condition (6).

We claim that for any graph $g$, if a player contemplates breaking a link, yielding $g^{\prime}$, the change of her payoff (i.e., her Myerson value) will only depend on the worth of the coalitions that are connected in $g$ and not connected in $g^{\prime}$. To see this, consider the value of player $i$ for a cooperation structure $g$ and $g \backslash\{i: j\}$.

$$
\begin{aligned}
\mu_{i}(v, g) & =\sum_{S \ni i} \rho_{S}((v / g)(S)-(v / g)(S \backslash i)) \\
\mu_{i}(v, g \backslash\{i: j\}) & =\sum_{S \ni i} \rho_{S}((v / g \backslash\{i: j\})(S)-(v / g \backslash\{i: j\})(S \backslash i)),
\end{aligned}
$$

where $\rho_{S}=\frac{(n-1) !(n-s) !}{n !}$. Notice that

(i) $g /(S \backslash\{i\})=(g \backslash\{i: j\})(S \backslash\{i\})$,

(ii) $(g \backslash\{i: j\}) / S \neq g / S$ when $i: j$ connects $S$ in $g$, i.e., when $S \in \mathbf{S}(i: j)$,

(iii) $(g \backslash\{i: j\}) / S=g / S$ when $i: j$ does not connects $S$ in $g$.

Using (3) we deduce

(a) (i) $\Rightarrow(v / g)(S \backslash\{i\})=(v / g \backslash\{i: j\})(S \backslash\{i\}), \forall S \ni i$,

(b) (ii) $\Rightarrow(v / g \backslash\{i: j\})(S) \neq(v / g)(S)$ when $S \in \mathbf{S}(i: j)$,

(c) (iii) $\Rightarrow(v / g \backslash\{i: j\})(S)=(v / g)(S)$ when $S \notin \mathbf{S}(i: j)$, 
Thus,

$$
\begin{gathered}
\mu_{i}(g)-\mu_{i}(g \backslash\{i: j\}) \geq 0 \\
\Leftrightarrow \sum_{S \in \mathbf{S}(i: j)} \rho_{S}\left(v(S)-v\left(S_{i}\right)-v\left(S_{j}\right)\right) \geq 0,
\end{gathered}
$$

which proves the claim. Observe that if $g=g_{N}$, then $\mathbf{S}(i: j)=\{\{i, j\}\}$. In other words, in the full cooperation structure, if a player $i$ breaks a link with player $j$, the only coalition that becomes non-connected is $\{i, j\}$.

More generally, suppose that some player $i$ severs her links in $g_{N}$ with players $j \in S$, where $S$ is some subset of $N \backslash\{i\}$. Then we have $\{\mathbf{S}(i: j)\}_{j \in N}=$ $\left\{\{i, j\}_{j \in S}\right\}$. This implies that $i$ severs her links with players $j \in S \subseteq N \backslash\{i\}$ if, and only if

$$
\sum_{j \in S} \rho_{\{i j\}}(v(\{i j\})-v(\{i\})-v(\{j\}))<0 .
$$

As (8) holds for any $S \subseteq N \backslash\{i\}$, for any $i \in N$, the result follows.

Qin (1996) showed that if the underlying TU-game is superadditive, then for any partition $\mathscr{P}$ of $N$, there is a Nash equilibrium $\sigma$ game such that $N / g(\sigma)=\mathscr{P}$ - see Qin (1996, remark 2). We now show that this result can be extended. ${ }^{5}$

Proposition 2 Let $(N, v)$ be a superadditive TU-game. For any $g \subseteq g_{N}$, there exist a strategy profile $\sigma$ such that $g=(\sigma)=g$ and $\sigma$ is a Nash equilibrium of $\mathscr{G}_{\mu}^{(N, v)}$.

Proof Consider any superadditive game $(N, v)$ and any cooperation structure $g$. Hence, (7) always holds and we can deduce that no player wishes to break a link. Consider the following strategy profile. For each player $i \in N$, let $\sigma_{i}(j)=0$ if $i: j \notin g$ and $\sigma_{i}^{*}(j)=1$ if $i: j \in g$. Because players do not sever links, if a player $i$ deviates with the strategy $\hat{\sigma}$, it should be the case that

\footnotetext{
${ }^{5}$ See Dutta, van den Nouweland, and Tijs (1998, proposition 1) for a similar result.
} 
for some $j$ we have $\hat{\sigma}_{i}(j)=1$ and $\sigma_{i}^{*}(j)=0$. But deviations are individuals, which implies that $\sigma_{j}^{*}(i)=0$. Hence, $g\left(\hat{\sigma}_{i}, \sigma_{-i}^{*}\right)=g$, and player's $i$ payoff remains unchanged. Thus, $i$ has no incentives to deviates. 


\section{References}

Aumann, R. J., And R. B. Myerson (1988): "Endogenous Formation of Links between Players and of Coalitions: an Application of the Shapley Value," in The Shapley value, ed. by A. Roth, pp. 175-191. Cambridge Univ. Press, Cambridge.

BollobÁs, B. (1998): Modern graph theory. Springer-Verlag, New York.

Dutta, B., A. van den Nouweland, and S. Tijs (1998): "Link Formation in Cooperative Situations," International Journal of Game Theory, $27,245-256$.

Jackson, M. O., And A. Wolinsky (1996): "A Strategic Model of Social and Economic Networks," Journal of Economic Theory, 71, 44-74.

Myerson, R. B. (1977): "Graphs and Cooperation in Games," Mathematics of Operation Research, 2, 225-229.

QIN, C.-Z. (1996): "Endogeneous Formation of Cooperation Structures," Journal of Economic Theory, 69, 218-226. 